\title{
Manipulating quantum paths for novel attosecond measurement methods
}

\author{
Kyung Taec Kim, D. M. Villeneuve and P. B. Corkum ${ }^{\star}$
}

\begin{abstract}
Sources of attosecond-duration light pulses provide the fastest time resolutions available today for observing ultrafast phenomena in atoms, molecules and condensed matter. The measurement of such pulse durations is challenging because the spectrum lies in the vacuum ultraviolet or soft X-ray range. Two classes of pulse duration measurements now exist; they are classified according to whether the measurement is performed in the generating medium or in a second medium. The first measurement class is called 'in situ' and depends on gently perturbing the electron responsible for attosecond pulse formation. The second measurement class, which we refer to as 'ex situ', takes place in a second medium in which photoelectrons are produced by the attosecond pulses; a synchronized laser field perturbs the photoelectron spectrum. This Review compares and contrasts these two approaches for measuring attosecond pulses.
\end{abstract}

S everal new approaches to studying the structure of matter on very fast timescales have emerged in the past few decades. Brief pulses of electrons are used to perform ultrafast diffraction of molecules ${ }^{1}$ and condensed matter ${ }^{2}$. Femtosecond lasers have been employed to observe chemical dynamics ${ }^{3}$. X-ray free-electron lasers generate intense bursts of X-rays that can be used to determine the structure of biomolecules ${ }^{4,5}$. Attosecond pulses in the vacuum ultraviolet, extreme ultraviolet or soft X-ray range are the shortest of all current probe pulses. Such pulses have moved the time resolution range from the femtosecond scale (the timescale for atomic motion in molecules) to the attosecond scale (the timescale for electron motion in atoms). The atomic unit of time is 24 as, and a semiclassical electron orbits a hydrogen atom in 150 as. The shortest individual attosecond pulses generated to date have a duration of 67 as (ref. 6).

The process of high-harmonic generation (HHG) underlies the production of attosecond pulses ${ }^{7-10}$; it is described by the so-called three-step mode ${ }^{11-13}$. The simplest description of HHG is a classical one. The laser field detaches an electron from a gas-phase atom. The electron is then accelerated by the field, and photorecombines with its parent ion. On recombination, the kinetic energy of the electron is released in the form of a single photon. The entire process takes place within a single optical cycle, and results in the production of a single attosecond pulse. If the driving laser field contains many optical cycles, then a train of attosecond pulses will be produced. The emission is coherent and can be phase matched among many emitting atoms ${ }^{14-16}$, leading to coherent and collimated emission of attosecond pulses. If we are careful about phase matching, the measured signal will be an amplified version of the single-atom response.

Ultrafast temporal dynamics in atoms and molecules can be probed by attosecond pulses. An atom irradiated by an attosecond pulse produces a continuum photoelectron wave packet whose amplitude and phase are determined by the amplitude, phase and polarization of the attosecond pulse, as reflected by the transition moment ${ }^{17,18}$. If the atomic transition moment can be accounted for, then the characteristics of the photoelectron will uniquely encode the characteristics of the attosecond pulse that created it. Adding a weak laser pulse will modify the photoelectron replica ${ }^{19,20}$. It is this modification that allows us to measure the time structure of the photoelectron replica. Specifically, by scanning the phase relative to the attosecond pulse, we gain information about the emission time of the photoelectron replica. This frequency-resolved optical gating (FROG) concept allows us to fully characterize the electron wave packet, and hence the attosecond pulse $\mathrm{e}^{19,21-23}$.

The other approach for the measurement of attosecond pulses is to observe the pulse generation process itself. The trajectory of the electron that recombines can be gently controlled by an additional laser field. By controlling the additional field, it is possible to determine the space-time characteristics of the attosecond pulse in the medium as the pulse is being produced. We call this approach an 'in situ' measurement, as opposed to the 'ex situ' measurement, which utilizes a second atomic medium for the measurement.

The aim of this Review is to demonstrate the parallels between attosecond measurements based on photoionization (ex situ) and photorecombination (in situ). As attosecond measurements based on photoionization have been discussed elsewhere ${ }^{9,23}$, we will put more emphasis on in situ measurements based on photorecombination. Attosecond measurements based on photoionization and photorecombination are conceptually very similar. However, each biases us to think in a different manner; photoionization inclines us to consider the photon as the probe and to draw parallels with time-resolved photoelectron spectroscopy, whereas photorecombination predisposes us to view the electron as a probe and to draw parallels with electron diffraction and other collision experiments. Viewed together, we realize that attosecond diagnostics will allow us to simultaneously resolve both structure on the ångström scale and dynamics on the attosecond scale ${ }^{7}$. In addition, as the photorecombination underlying attosecond pulse generation takes place between correlated electrons and ions, attosecond science gives us unrivalled access to correlated dynamics.

\section{High-harmonic spectroscopy}

Three-step model. As mentioned above, the process of HHG is described by the so-called three-step model ${ }^{11,12}$. In the semi-classical description of HHG, the laser field detaches the electron, the electron is accelerated by the field, and the electron photorecombines with the parent ion. The classical equations of motion are written for an electron with mass $m$ and charge $e$ under the influence of an oscillating electric field $E(t)$ that represents the intense laser pulse, with the acceleration being $a(t)=(e / m) E(t)$. The acceleration can be integrated to give the velocity $v(t)$ and the position of the electron $x(t)$. The potential of the ion is neglected. 
The trajectories are shown in Fig. 1. Each trajectory is defined by the instant at which it becomes free of the atom, often called the time of birth, $t$. Trajectories that start before the peak of the laser field cycle do not return to the parent ion, whereas trajectories that begin after the peak will return to the ion, where they have a possibility of recolliding and recombining. In Fig. 1, the velocity at which they recollide is determined by the slope of each trajectory at zero displacement; the kinetic energy at recollision is denoted by the colour of each trajectory, with blue indicating the highest kinetic energy. If the electron recombines with the parent ion, it will emit a photon that has an energy equal to the kinetic energy of the electron plus the binding potential, $I_{\mathrm{p}}$. The most energetic trajectory will emit a photon whose energy is $\hbar \Omega=3.17 U_{\mathrm{p}}+I_{\mathrm{p}}$, where $U_{\mathrm{p}}=e^{2} E_{0}^{2} /\left(4 m \omega_{0}^{2}\right), \hbar$ is the reduced Planck constant, $E_{0}$ is the peak electric field and $\omega_{0}$ is the angular frequency of the laser field. The instantaneous photon energy first chirps up to the highest energy, then chirps back down; this chirp is known as the atto-chirp ${ }^{24-26}$.

The three-step model can be described quantum mechanically by the time-reversed $S$-matrix method ${ }^{27}$, also called the strong-field approximation (SFA).

$$
d(t)=-\mathrm{i} \int d k \int_{0}^{t} d t^{\prime}\langle\psi|r| k-A(t)\rangle \cdot \mathrm{e}^{-\mathrm{i} S} E\left(t^{\prime}\right) \cdot\left\langle k-A\left(t^{\prime}\right)|r| \psi\right\rangle
$$

Here, $d$ is the dipole response of the atom, $k$ is the canonical momentum, $A$ is the vector potential of the laser field, $\psi$ is the Dyson wavefunction describing the bound electron in the atom and $S$ is the classical action, which is defined as follows.

$$
S=\int_{t^{\prime}}^{t} d t^{\prime \prime}\left(k-A\left(t^{\prime \prime}\right)\right)^{2} / 2+I_{\mathrm{p}}\left(t-t^{\prime}\right)
$$

Here, $I_{\mathrm{p}}$ is the ionization potential. Reading equation (1) from right to left, ionization occurs at time $t$ ', the electron accumulates a Volkov phase in the continuum (described by the classical action $S$ ) and recombines back to $\psi$ at time $t$. The radiated electromagnetic spectrum ${ }^{28}$ is the square of the Fourier transform of the second derivative of $d(t)$ :

$$
I_{\Omega}(\Omega)=\Omega^{4}\left|\int d(t) e^{-\mathrm{i} \Omega t} d t\right|^{2}
$$

The results of the classical three-step model can be approximately reproduced by the SFA model by finding the stationary points of the classical action $S$; this approximation predicts that the electron must originate and terminate near the ion.

Equation (1) describes the single-atom part of the HHG process. Because this process begins and ends with the atom in the state described by wavefunction $\psi$, the phase of $\psi$ cancels out. This means that the process is coherent, being independent of the phase of the atom, which permits the macroscopic emission to be phase matched. Non-phase-matched emission radiates into $4 \pi \mathrm{sr}$ and is usually not detected experimentally. Thus, HHG is very sensitive to the wavefunction $\psi$ of the atom and discriminates against other processes that do not end at the initial state.

Perturbation of electron trajectories. In the HHG process, the electron moves along specific trajectories under the influence of the fundamental driving laser field, from the time of birth $t$ ' to the recombination time $t$. During this excursion, the electron accumulates a phase that is transferred to the HHG radiation. Any

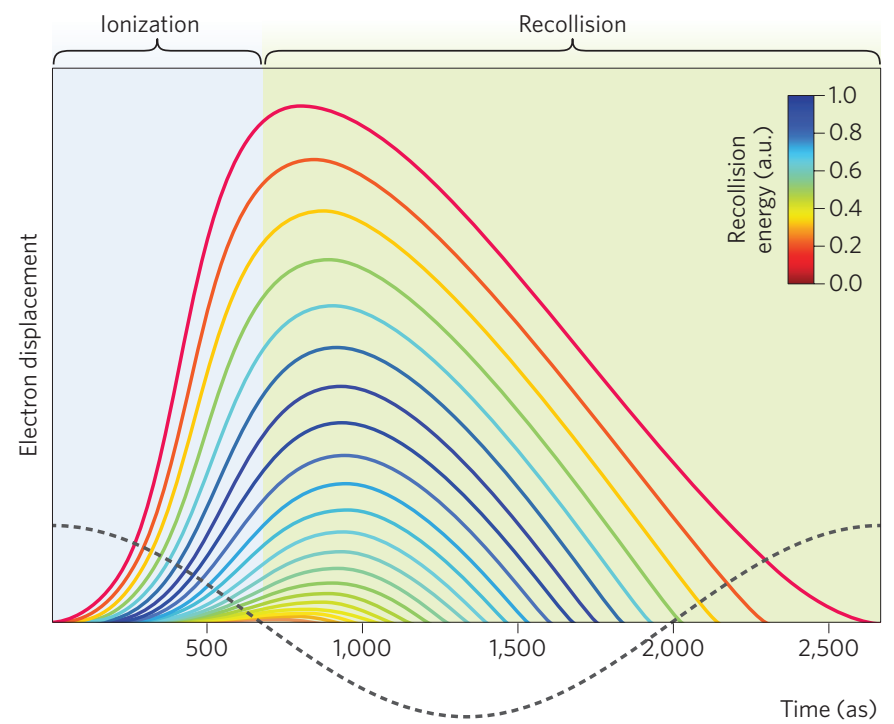

Figure 1 | Electron trajectories involved in high-harmonic generation. The instantaneous electric field of the laser is denoted by the dashed line. Individual electron trajectories that return to the origin are shown for various times after the peak of the field. The velocities at which they return are given by the slopes of the trajectories at the origin. The colour scale indicates the relative kinetic energy on return. The different electron trajectories can recombine at a range of times between 700 as and 2,500 as after the field peak, leading to the emission of an attosecond pulse that chirps up and then down in frequency. This process repeats every half optical cycle, leading to the emission of a train of attosecond pulses. Figure reproduced with permission from ref. 69 (c) 2012 NPG.

perturbation made to the laser field modifies the electron trajectory, and similarly changes the phase of the HHG radiation.

The phase shift $\sigma_{q}$ at the $q$ th harmonic order imposed by the addition of a perturbing field $E_{\mathrm{p}}(t)$ can be derived from the classical action shown in equation (2) as

$$
\sigma_{q}(\tau)=-\int_{t^{\prime}(t)}^{t} d t^{\prime \prime}\left[\mathbf{v}_{\mathrm{SFA}}\left(t^{\prime \prime}\right) \int_{t^{\prime}(t)}^{t^{\prime \prime}} d t^{\prime \prime \prime} E_{p}\left(t^{\prime \prime \prime}-\tau\right)\right]
$$

Here, $\tau$ is the relative delay between the fundamental driving field and the perturbing field, and $\mathbf{v}_{\mathrm{SFA}}$ is the unperturbed electron velocity ${ }^{29,30}$.

Unlike other nonlinear processes, such as second-harmonic generation in a nonlinear crystal, the HHG process is temporally localized. The electron trajectory that generates the $q$ th harmonic radiation is born at $t^{\prime}$ and recombines at $t$. The electron experiences the additional field only during the excursion time (from $t^{\prime}$ to $t$ ). As we show below, this temporally localized perturbation effect allows us to probe the dynamics of the attosecond pulse generation process itself and the perturbing field. Here, we reveal how a small field can perturb the electron trajectories, leading to a significant phase shift.

In $\mathrm{HHG}$, the ionization-recollision process repeats every half optical cycle, leading to the generation of an attosecond pulse train. The electron recollides from alternate sides of the atom during alternate half optical cycles (Fig. 2a). This leads to a series of attosecond pulses that chirp up and down in frequency. If the spectrum of each attosecond pulse is $F(\omega)$, then by the Fourier repetition property the spectrum of the pulse train will be $F(\omega) \Sigma_{n} \delta\left(\omega-(2 n+1) \omega_{0}\right)$ (that is, the spectrum is modulated into a series of odd harmonics of the driving laser field $\omega_{0}$ ). The harmonics are odd rather than even 
a
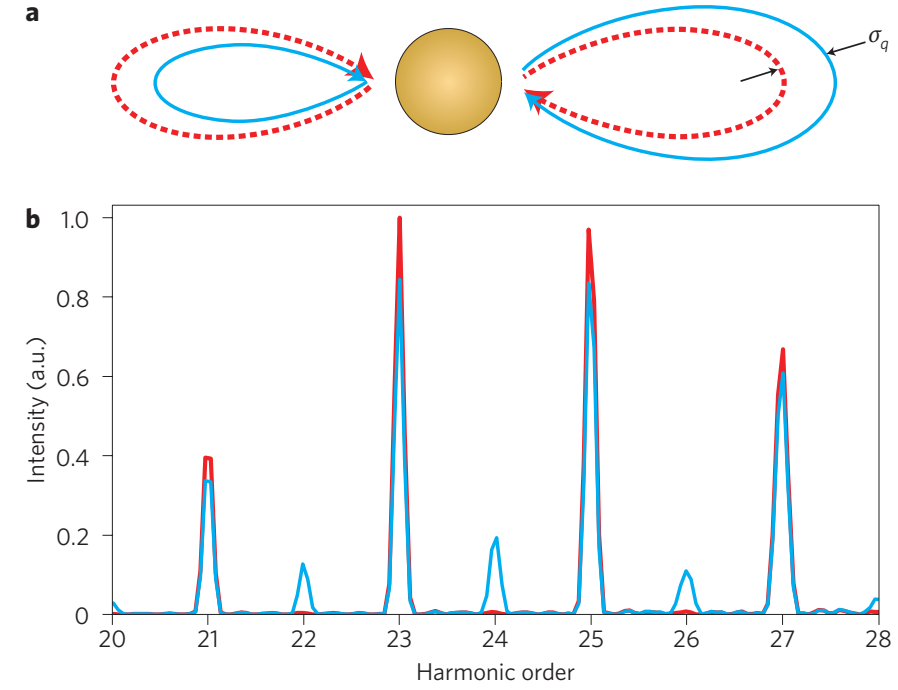

Figure $\mathbf{2}$ | Second-harmonic field leads to even-harmonic emission. $\mathbf{a}$, The addition of a weak second harmonic of the fundamental laser frequency affects the electron trajectories differently for alternating half optical cycles, as shown by the blue lines. One trajectory is slightly longer than the other, leading to a phase shift of the Volkov phase for the trajectory. b, By breaking the strict inversion of alternating attosecond pulses, the secondharmonic field causes the production of even harmonics of the fundamental laser frequency, in addition to the usual odd-harmonic spectrum. The highharmonic spectrum is calculated from a single $\mathrm{Ne}$ atom with (blue line) and without (red line) the perturbing second-harmonic field. The fundamental and perturbing second-harmonic pulses have intensities of $3 \times 10^{14} \mathrm{~W} \mathrm{~cm}^{-2}$ and $1.5 \times 10^{11} \mathrm{~W} \mathrm{~cm}^{-2}$, respectively.

because of the alternating parity of each attosecond pulse; emission at even harmonics is completely cancelled.

If the laser field includes even a weak component at a different frequency, the exact alternation of the pulse train is broken, leading to the possibility of generating other frequencies besides those of the odd harmonics. For example, a second-harmonic field $2 \omega_{0}$ will make the positive and negative cycles of the fundamental field different, leading to the appearance of even harmonics. Figure 2 illustrates how a weak second-harmonic field can perturb the electron trajectories for alternate half cycles. The HHG spectrum calculated from a single $\mathrm{Ne}$ atom with an intensity of $3 \times 10^{14} \mathrm{~W} \mathrm{~cm}^{-2}$ is shown by the red line in Fig. 2 b. The second-harmonic pulse that has only $0.05 \%$ of the fundamental intensity is strong enough to perturb the HHG process, leading to the formation of even-order harmonics (Fig. 2b). This feature of adding a second-harmonic field can be used to diagnose the process leading to the production of attosecond pulses, as will be discussed below.

Relationship between photoelectron and high-harmonic spectroscopy. Although the three-step model explicitly includes the bound-state wavefunction of the electron that was removed by the laser field (or more precisely, the Dyson orbital that corresponds to the difference between the neutral and the ion) through the recombination dipole moment from the continuum wavefunction to the bound wavefunction, it is not obvious how this dependence manifests itself in the HHG spectrum, which is the usual observable. It has indeed been shown that the HHG three-step process can be factorized into three steps, not just in the time domain, but also in the frequency domain ${ }^{31-34}$. From ref. 34, the power spectrum $I_{\Omega}\left(E_{\Omega}\right)$ can be approximately factored as follows.

$$
I_{\Omega}\left(E_{\Omega}\right)=I(F, \omega) W(E) \sigma^{\mathrm{r}}(E)
$$

Here, the term $I$ represents the ionization step, $W$ represents the acceleration of the electron in the continuum and $\sigma^{\mathrm{r}}$ is the photorecombination cross-section. $E$ is the kinetic energy of the recolliding electron, and $E_{\Omega}=E+I_{\mathrm{p}}$. The approach used by Itatani et al. ${ }^{31}$ was to determine the product of $I$ and $W$ using a reference atom for which $\sigma^{\mathrm{r}}$ is known. Then, $I_{\Omega}$ is a measure of $\sigma^{\mathrm{r}}$ for the target atom or molecule, the basis of quantitative rescattering theory ${ }^{35-38}$.

Consider the diagrams in Fig. 3. The first diagram shows the process of single-photon photoionization ${ }^{39}$. If the photon has sufficient energy, it can remove an electron from the atom. As the photon energy is increased, more-deeply bound electrons can be removed. Because the valence electrons are responsible for most of the chemical properties of a molecule, it is desirable to employ photon energies just above the ionization potential so as to remove only a valence electron. When an electron has been removed, the ion must end up in an eigenstate; the electron removes any excess energy as kinetic energy. The field of photoelectron spectroscopy ${ }^{40}$ uses this approach, by measuring the energy and direction of photoelectrons.

Figure $3 \mathrm{~b}$ shows the process of HHG, as described above. The electron is removed by an intense laser field by the electron a Photoionization

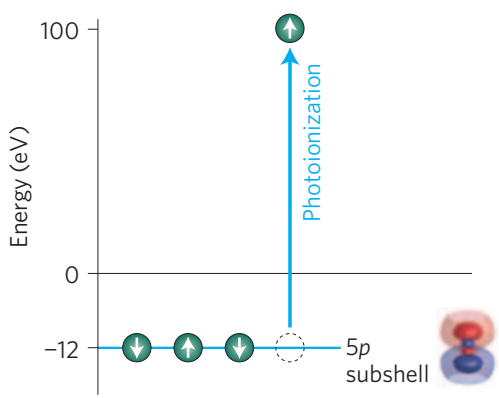

b High-harmonic generation

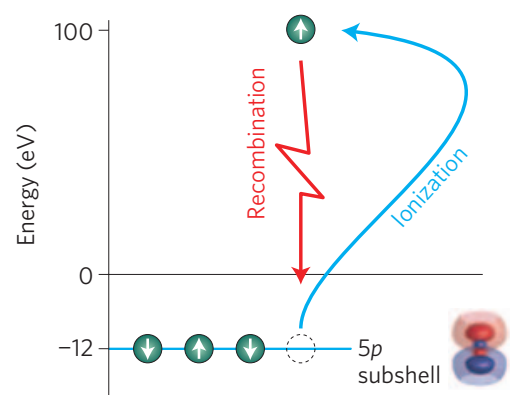

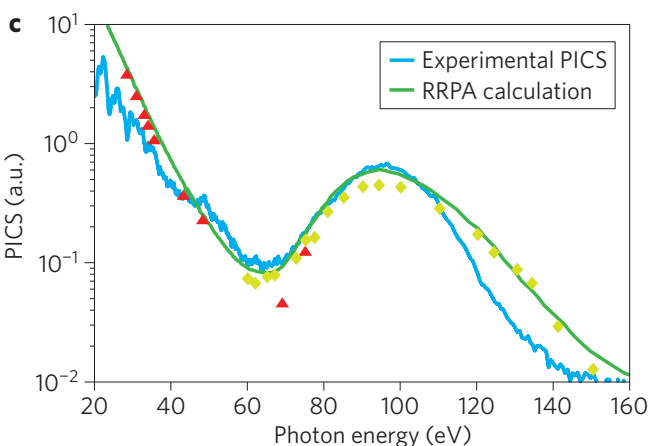

Figure 3 | Photoionization vs. high-harmonic generation. a, Energy diagram showing how a single photon can ionize an atom. The electron kinetic energy takes up the difference in energy between the photon energy and the binding energy of the electron. This is the basis of photoelectron spectroscopy. b, Illustration of the high-harmonic process. A valence electron is tunnel ionized by the laser field, which subsequently causes it to recollide with the parent ion. The electron can recombine, emitting a photon in the process. c, The HHG spectrum from xenon atoms extends to $160 \mathrm{eV}$, illustrating the broad range of frequencies obtained simultaneously in $\mathrm{HHG}$ experiments. The blue line is the HHG experiment ${ }^{52}$. The green line is a calculation of the photoionization cross-section ${ }^{53}$, and the symbols are synchrotron measurements ${ }^{54,55}$. PICS, photoionization cross-section; RRPA, relativistic random-phase approximation. Figure c reproduced with permission from ref. 52 (c) 2011 NPG. 


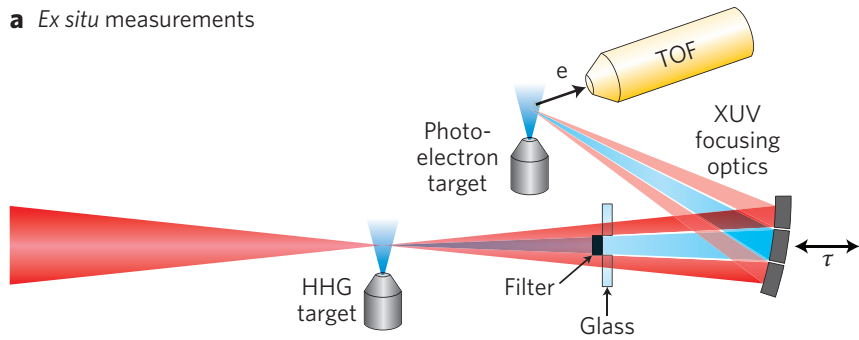

b In situ measurements

Figure 4 | Exsitu and in situ measurements. a, A typical experiment set-up for ex situ measurement. The attosecond pulses are generated in the HHG target, and refocused into the photoionization target. The laser pulse and the attosecond pulses are separated by an X-ray filter mounted on an annular glass pellicle. The time delay is controlled by moving the inner mirror. TOF, time-of-flight spectrometer. $\mathbf{b}, \mathrm{A}$ typical experiment set-up for insitu measurements. The perturbing beam can be spatio-temporally overlapped with the fundamental driving laser beam. The HHG spectrum is measured with a standard extreme-ultraviolet spectrometer that has a slit, extreme-ultraviolet grating and extreme-ultraviolet imaging detector.

tunnelling through the potential barrier ${ }^{41}$. This step is exponentially sensitive to the binding energy of the electron ${ }^{41}$, and so, unlike single-photon photoionization, is not able to remove deeply bound electrons. The recombination process is a timereversed version of photoionization, as the continuum wavefunction recombines through a dipole transition back to the ground state. For both processes, the transition matrix element between the bound and continuum states is the same. Despite the fact that in HHG the returning electron looks like a plane wave as it approaches the ion, it is now thought that the continuum wavefunction can be described as a scattering state in both cases ${ }^{32}$. Equation (4) shows that the HHG spectrum is proportional to the photorecombination cross-section, which is proportional to the photoionization cross-section.

This correspondence between photoionization and photorecombination has led to the field now called high-harmonic spectroscopy ${ }^{31,42-46}$. High-harmonic spectroscopy can provide complementary information to photoelectron spectroscopy, and has some advantages over it. For example, because the laser pulses are 5-30 fs in duration, impulsive alignment ${ }^{47-49}$ can be used to provide aligned molecules, permitting molecular-frame measurements to be made. The emitted HHG photons can be characterized by energy, polarization and phase, providing important information about the transition matrix ${ }^{50,51}$. Unlike photoelectron spectroscopy, high-harmonic spectroscopy utilizes a wide band of photon energies simultaneously.

Figure $3 \mathrm{c}$ shows a HHG spectrum from xenon atoms, using a $1.8 \mu \mathrm{m}, 12$ fs laser ${ }^{52}$. The green line indicates the calculated photoionization cross-section of xenon ${ }^{53}$ and the symbols show the cross-sections measured in synchrotron experiments ${ }^{54,55}$. The HHG spectrum shows the same features, demonstrating the parallels between photoionization and photorecombination. The large bump at $100 \mathrm{eV}$ is the well-known giant plasma resonance in xenon, a manifestation of electron-electron correlations that is faithfully reproduced by the HHG experiment.

It is remarkable that the HHG spectrum is a measure of the photoionization (or photorecombination) cross-section, as the recombination occurs in the presence of an intense laser field. It is important to be aware of this when performing spectroscopic experiments. For example, in experiments where holes are produced in a molecule $e^{50}$, the electron dynamics may be influenced by the laser field. In addition, there is a subtle difference between in situ and ex situ measurements in how the transition dipole is included. Because in situ measurements operate by perturbing the electron trajectory, they are not sensitive to the transition dipole. On the other hand, ex situ measurements record the electromagnetic pulse after it has exited the medium, and hence they are influenced by the transition dipole.

\section{Ex situ and in situ measurements}

After the first experimental observation of high-harmonic emission $^{56}$, many theoretical studies suggested the underlying formation of attosecond pulses ${ }^{57-59}$. Because attosecond pulses can be used as a coherent light source that provides an unprecedented temporal resolution for pump-probe experiments, attosecond-pulse measurement quickly became a popular area of research. However, there is no efficient nonlinear material in the photon energy range of HHG radiation. Instead, nonlinear interactions using photoionization, or the HHG process itself, are utilized for the attosecond pulse measurement.

In ex situ attosecond pulse measurements using the photoionization process, the attosecond pulse is employed as a light source for pump-probe-type experiments ${ }^{17,21,22,24,60-66}$. Figure $4 \mathrm{a}$ shows a typical experimental set-up for the ex situ measurement. In the first gas target, attosecond pulses are generated through HHG by focusing the strong laser field with an intensity range of $10^{13}$ $10^{15} \mathrm{~W} \mathrm{~cm}^{-2}$. The laser pulse is separated from the generated attosecond pulses; in Fig. 4a, an X-ray filter mounted on a glass pellicle is shown as an example. The attosecond pulse transmitted through the filter is refocused on the second target to produce the photoelectron. The laser pulse transmitted through the glass pellicle is also refocused on the second target with a time delay. The photoelectrons measured in the presence of the time-delayed laser field are used as a signal to measure the attosecond pulses. As we explain below, the reconstruction of attosecond beating by interference of two-photon transitions (RABBITT) technique ${ }^{17,24,26}$ and the attosecond streak camera ${ }^{21,22,24,60-64}$ are ex situ measurements in which the generated attosecond pulses are independently measured at a different location.

In an alternative approach to measurement, the re-colliding electron in HHG can be used to measure the attosecond pulses. In the HHG experiment, a weak perturbing field is added collinearly ${ }^{29,67}$, non-collinearly ${ }^{30,68}$ or collinearly with orthogonal polarizations ${ }^{69}$ to probe the dynamics of the attosecond pulse generation process. The HHG spectrum measured with the time-delayed perturbing field is the signal for the attosecond pulse measurement, and can be measured with an all-optical experimental set-up (Fig. 4b). These are

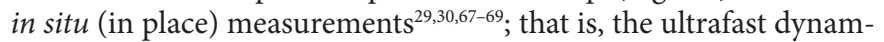
ics of attosecond pulse generation in atoms or molecules is studied at the place where it is generated.

Although these two approaches - ex situ and in situ - seem very different from each other, they are fundamentally linked. In both approaches, the additional laser field plays a critical role, and acts as a temporal gate. The phase of the attosecond pulses and the gate field are retrieved from the photoelectron spectrum (ex situ) or the HHG spectrum (in situ) modulated by a time-delayed gate pulse. This FROG-like idea allows us to probe the dynamics of the 


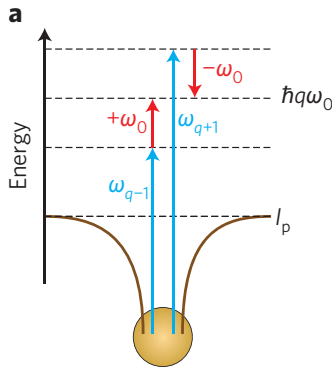

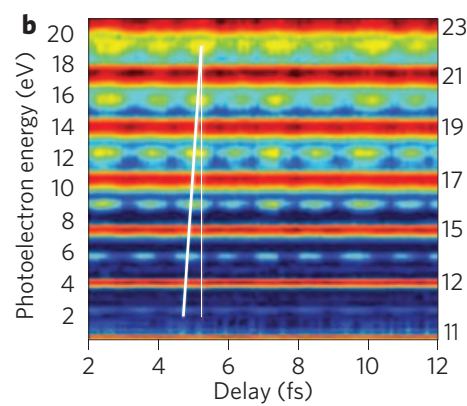

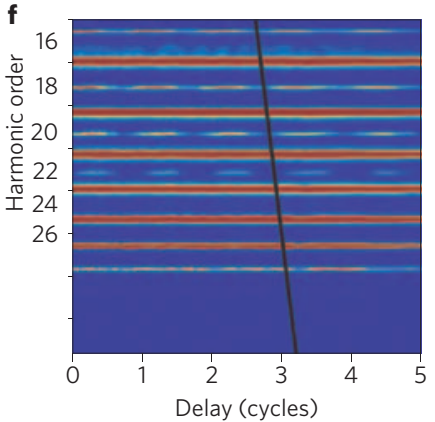

Delay (cycles)
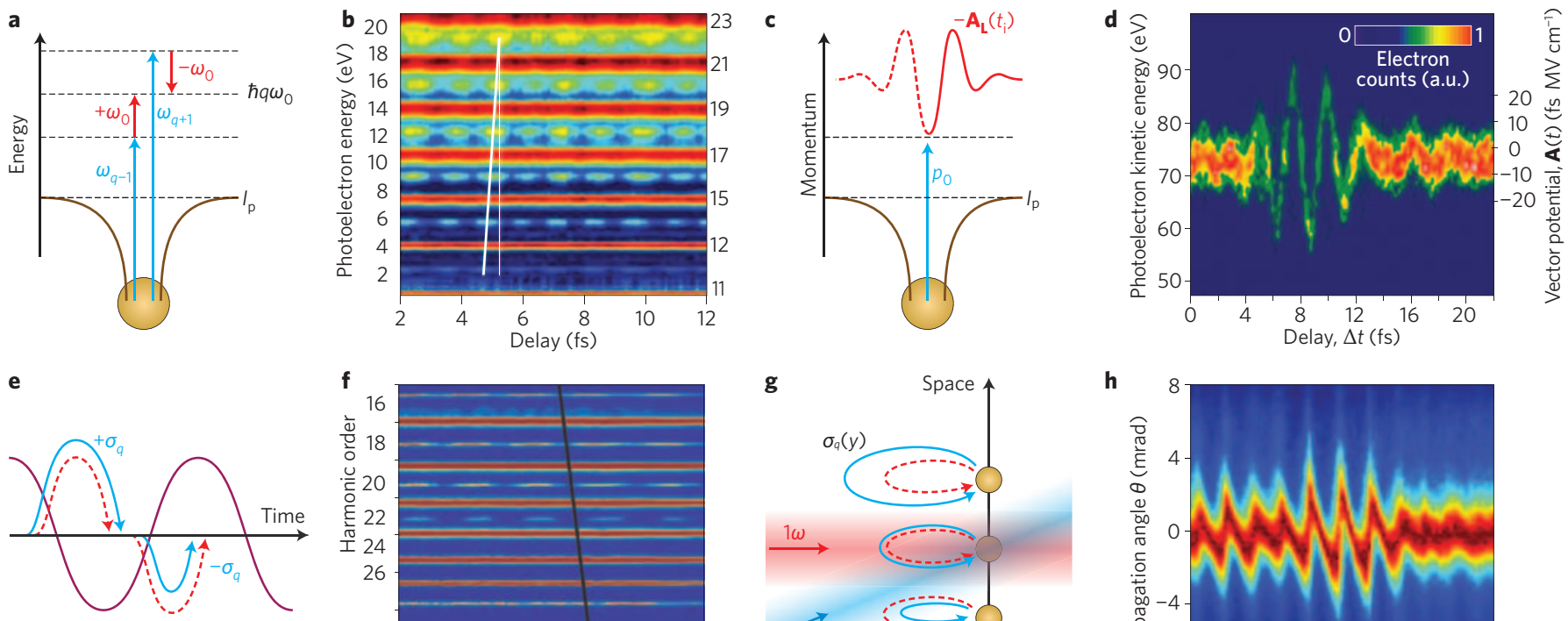

g

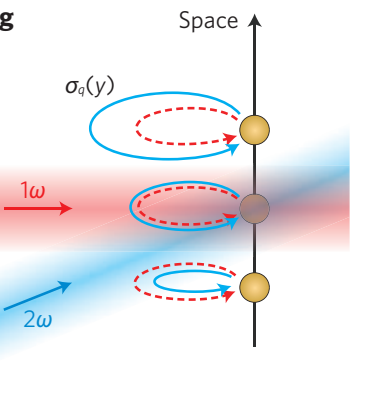

h

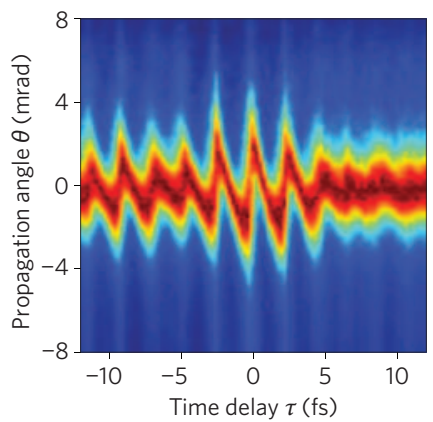

Figure $\mathbf{5}$ | Attosecond pulse and optical field measurements with ex situ and in situ measurements. a, A schematic transition diagram for the formation of the even-order sideband through a two-photon transition in the RABBITT measurement. $\mathbf{b}$, A photoelectron spectrum measured with the attosecond pulse train in the RABBITT experiment. c, A schematic showing the momentum shift of the photoelectron in FROG CRAB measurements. d, Photoelectron spectrum measured with an isolated attosecond pulse in a FROG CRAB experiment. e, A schematic showing the electron trajectories in collinear in situ measurement. The original (red dotted line) and perturbed (blue lines) electron trajectories are shown with the fundamental driving laser field (thin red line). f, High-harmonic spectrum with a weak second-harmonic field in collinear in situ measurement. $\mathbf{g}$, The original (red dotted lines) and perturbed (blue lines) electron trajectories of electrons at different positions across the focus in the STRAP measurement. $\mathbf{h}$, The angular distribution of the harmonic radiation in the petahertz optical oscilloscope ${ }^{68}$. Figure reproduced with permission from: b, ref. 24, (c) 2003 AAAS; d, ref. 62, (c) 2004 AAAS; f, ref. 29, (c) 2006 NPG; h, ref. 68 (c) 2013 NPG.

attosecond pulse generation as well as the optical field of the additional laser field. In the next section, we review the two approaches, emphasizing the measurement of attosecond pulses, optical fields and electron birth times.

Reconstruction of attosecond beating by interference of twophoton transitions. The first experimental observation of attosecond pulses was carried out by the ex situ technique ${ }^{17}$ involving the RABBITT. In the RABBITT technique, the attosecond pulse train consisting of odd-order harmonics produces discrete photoelectron energies with a separation of $2 \omega_{0}$. As the photoelectron spectrum is the replica of the attosecond pulse, only odd-order harmonics would appear without the additional laser field. However, continuum-continuum transitions occur due to the presence of the additional laser field, resulting in the formation of spectral sidebands ${ }^{70}$. Figure 5a shows the transition diagram. The sideband with energy $\hbar q \omega_{0}$, where $q$ is even, may be produced through two different quantum paths, leading to the possibility of interference.

As a result of the interference between the two quantum paths, the amplitude of the sideband oscillates as a function of the time delay $\tau$ between the attosecond pulse and the laser pulse (Fig. 5b). This oscillation occurs because $S_{q} \propto \sin \left[2 \omega_{0}\left(\tau-\tau_{q}^{\mathrm{GD}}-t_{q}^{\text {atom }}\right)\right]$, where $\tau_{q}{ }^{\mathrm{GD}}$ is the group delay near the $q$ th harmonic. Assuming we know the intrinsic atomic delay $t_{q}^{\text {atom }}$ imposed in the photoionization pro$\operatorname{cess}^{17,70}$, the group delay (or emission time) of each harmonic can be determined. The slope that connects the maxima of the sidebands (indicated by the thick white line in Fig. 5b; the thin white line shows the vertical direction) represents the group delay difference of each harmonic, known as the 'atto-chirp ${ }^{24-26}$. This phase information can be combined with the amplitude of harmonics to reconstruct the temporal profile of the attosecond pulse train. Because this technique requires a comb-like spectrum, it is applicable only for attosecond pulse trains; it cannot be applied for isolated attosecond pulses.

Attosecond streak camera. The advent of carrier-envelope-phasestabilized few-cycle pulse laser sources enabled the generation of isolated attosecond pulses ${ }^{71,72}$. Several different approaches for selecting an isolated attosecond pulse from the train have been employed, including high-frequency filtering ${ }^{22,58,61,72}$, polarization gating $^{21,59}$, double-optical gating ${ }^{73}$, ionization gating ${ }^{25,74,75}$ and photonic streaking ${ }^{76,77}$. The attosecond streak camera ${ }^{20}$ was developed to characterize isolated attosecond pulses. In the attosecond streak camera, an isolated attosecond pulse produces a photoelectron in the presence of an additional laser field $E_{\mathrm{L}}(t)$ (Fig. $5 \mathrm{c}$ ). The generated photoelectron is then driven by the additional laser field after the ionization time $\left(t_{\mathrm{j}}\right)$. The final momentum of the photoelectron is shifted by $-\mathbf{A}_{\mathbf{L}}\left(t_{\mathrm{i}}\right)$ where $\mathbf{A}_{\mathbf{L}}$ is the vector potential of the additional laser field (that is, $E_{\mathrm{L}}=-\partial \mathbf{A}_{\mathrm{L}} / \partial t$ ). The photoelectron spectrum measured with a time-delayed laser pulse shown in Fig. 5d is the measurement signal for an attosecond pulse. The temporal profile of the attosecond pulse is determined by a phase-retrieval algorithm known as frequency-resolved optical gating for the complete reconstruction of attosecond bursts (FROG CRAB) ${ }^{19}$.

Direct measurement of light field. In addition to measuring the attosecond pulse, the attosecond streak camera also directly measures the vector potential of the laser field ${ }^{19}$. This was the first direct observation of the subcycle electric field of a laser pulse in the visible range $^{62}$. Conventional ultrafast optical techniques commonly used to characterize visible light pulses (such as FROG ${ }^{78}$ and SPIDER $^{79}$ ) are frequency-domain measurements in which the relative phase of 
different Fourier components is measured. In addition, these conventional techniques suffer from the limited bandwidths of the nonlinear materials used in measurements. In contrast, the attosecond streak camera is a time-domain measurement. The momentum shift of the photoelectron directly reveals the oscillating light field itself (Fig. 5d). More precise analysis can be achieved using the FROG CRAB technique ${ }^{19}$. The temporal resolution of the measurement is limited only by the bandwidth of the attosecond pulse, which could go below 100 as. Therefore, the measurement covers the broad spectral ranges of the additional laser field in the ultraviolet, visible and infrared regions ${ }^{62,63}$.

Delay in photoionization. Ex situ techniques, including RABBITT and the attosecond streak camera, measure the emission time of the photoelectron produced by the attosecond pulse in the presence of an additional field. The ex situ techniques actually measure the amplitude and phase of the photoelectron ${ }^{80}$. Within the SFA, the phase shift imposed during photoionization has to be corrected using the calculated intrinsic atomic delay. After the photoionization, the electron in the additional field is classically treated without considering the effect of the ionic potential. However, recent measurements of the delay in photoionization from different orbitals could not be successfully explained by this analysis ${ }^{64}$. Using the FROG CRAB technique with an isolated attosecond pulse, a delay of $21 \pm 5$ as in the photoionization from the $2 p$ orbitals of $\mathrm{Ne}$ atoms with respect to those from the $2 s$ orbitals was measured. In addition, delays from -40 as to -110 as for the $3 s$ and $3 p$ orbitals of Ar atoms were measured using the RABITT technique with an attosecond pulse train ${ }^{65}$.

These measurements motivated several theoretical studies ${ }^{65,80-83}$, which claim that the delay in the ex situ measurements can be caused in both steps - the photoionization and the streaking. The linear phase variation of the transition moment causes a delay in the photoionization, known as the 'Wigner time delay'. In addition, the ionic potential affects the electron dynamics during the streaking, which cause another 'measurement-induced delay'. Based on this new interpretation, the expression for the sideband intensity can be written as $S_{q} \propto \sin \left[2 \omega_{0}\left(\tau-\tau_{q}^{\mathrm{GD}}-t_{q}^{\text {atom }}-\tau_{q}^{\mathrm{CC}}\right)\right]$, where $\tau_{q}^{\mathrm{CC}}$ is the delay imposed in the continuum-continuum transition during the streaking ${ }^{83}$. How these delays can be disentangled remains to be studied ${ }^{82}$.

Collinear in situ measurements. The in situ approaches measure temporal dynamics during the HHG process itself. In collinear in situ measurements, a weak second-harmonic field is collinearly superimposed with the fundamental laser pulse to perturb the HHG process $^{29}$. HHG repeats at every half optical cycle of the fundamental laser pulse along alternate directions. If the electron recombines along one side of the atom in one half cycle, it will recombine along the other side of the atom in the next half cycle (Fig. 5e). Because the electron moves with mirror symmetry for two consecutive half cycles (red dotted line), only odd-order harmonics are generated. The weak second-harmonic field breaks the mirror symmetry. The addition of the second-harmonic field slightly modifies the electron trajectories (blue lines). The phase of the attosecond pulse is modified by $+\sigma_{q}$ (equation (3)) during one half cycle and by $-\sigma_{q}$ during the next half cycle for the $q$ th harmonic (Fig. 5e). This asymmetric electron motion causes the formation of the even-order harmonics (Fig. 5f).

The addition of the second-harmonic field is effective only when the electron is in the continuum state from the ionization time to the recombination time. In the in situ measurements, this temporally localized perturbation effect is used as a temporal gate. The even-order harmonic is maximized when a peak of the secondharmonic field is well overlapped with the excursion of the electron from the ionization to recombination (Fig. 5f). Thus, the time delay that maximizes the even-order harmonics is linearly proportional to the recombination (or emission) time. This relationship is universal when the ionization potential is neglected ${ }^{29}$. Quantum mechanical approaches in which the ionization potential is taken into account suggest corrections when the intensity of the fundamental driving laser pulse is weak ${ }^{84}$.

Space-time reconstruction of attosecond pulses. Researchers further generalized the in situ measurement for characterizing isolated attosecond pulses ${ }^{30}$. As the collinear in situ measurement breaks the symmetry of two consecutive attosecond pulses in the time domain, it cannot be applied for isolated attosecond pulses. To measure isolated attosecond pulses, the second-harmonic beam is superimposed at a small angle relative to the propagation direction of the fundamental driving laser pulse. The additional second-harmonic beam serves as a spatial and temporal gate for the space-time characterization of the attosecond pulse; this method has been called space-time reconstruction of attosecond pulses $(\text { STRAP })^{30}$. Because of the small angle used in this technique, the time delay $\tau$ between the fundamental driving laser pulse and the weak second-harmonic beam varies depending on the transverse position $y$. The phase shift $\sigma_{q}(y)$ of the net driving field modifies the electron trajectories, and hence the wavefront of the attosecond pulse (Fig. 5g).

Any modification made in the near field changes the angular distribution of the attosecond pulse in the far field. The angular distribution measured as a function of the time delay $\tau$ shown in Fig. $5 \mathrm{~h}$ is a direct analogue of the photoelectron spectra shown in Fig. 5d. In the FROG CRAB technique, the additional field serves as a temporal phase modulator. The spectral amplitude and phase of the attosecond pulse are reconstructed using the photoelectron spectra. In STRAP, the additional second-harmonic field serves as a spatial phase modulator. The spatial amplitude and phase of the attosecond pulse is reconstructed from the spatial distribution at different photon energies. The phase shift $\sigma_{q}$ can also be found from the spatial distribution; it provides information on the group delay, which determines the phase relationship between different energies. Consequently, the amplitude and phase of the attosecond pulse can be completely characterized in space and time ${ }^{30}$.

Petahertz optical oscilloscope. In the in situ measurement, the phase shift is imposed by the additional field during the excursion time of the electron during the high-harmonic process. If the electron trajectory is shorter than the period of the perturbing field, it can be used as a fast temporal gate to probe the perturbing field. An arbitrary optical waveform $E_{\mathrm{p}}(t)$ can be superimposed at a small angle. The phase shift is proportional to the additional field (that is, $\left.\sigma_{q} \propto E_{\mathrm{p}}(\tau)\right)$, which modifies the wavefront of the harmonic radiation. Then, the propagation angle of the harmonic radiation is changed as $\Theta(\tau) \propto \mathrm{d} E_{\mathrm{p}}(\tau) / \mathrm{d} \tau$. The deflection angle of the harmonic radiation is proportional to the time derivative of the perturbing light field, permitting $E_{\mathrm{p}}(t)$ to be measured ${ }^{68}$ (Fig. $5 \mathrm{~h}$ ).

Resolving ionization and recombination time in HHG. The temporal dynamics of the electron in HHG starts at the ionization time and finishes at the recombination time. To resolve these two temporal events, two separate measurements are required. This is achieved by introducing an orthogonally polarized second-harmonic field with respect to the fundamental driving laser pulse ${ }^{69}$. The ionized electron is displaced by the orthogonal field; this 'displacement gate' suppresses the harmonic emission. The addition of the second-harmonic field also breaks the mirror symmetry of the electron motion in two consecutive half cycles. The symmetry breaking is maximized when the lateral velocity of the recombining electron is a maximum; this 'velocity gate' provides an additional 
independent observable. These two gates disentangle the ionization and recombination times of the electron in HHG. The reconstructed ionization and recombination times agree well with the real part of the complex ionization and recombination time obtained with the saddle-point approximation ${ }^{27}$ where the ionization potential is taken into account. Resolving ionization and recombination times opens up a way to observe the tunnel ionization process in more complicated systems ${ }^{69}$.

Applications for ex situ and in situ measurements. Both the ex situ and in situ approaches can be used to measure ultrafast dynamics. Ex situ measurements provide a completely independent measurement through photoionization in the presence of an additional laser field. They permit the attosecond pulses generated with single- or multicoloured laser pulses to be measured ${ }^{73,85}$. In addition, they can measure any modification made on the attosecond pulse after generation, such as absorption and dispersion, allowing the temporal profile of the attosecond pulse to be optimized. Such an optimized attosecond pulse $\mathrm{e}^{6,25,26}$ can be used to study the photoionization dynamics in atomic inner shells ${ }^{86}$ or solids ${ }^{87}$ and tunnelling dynamics ${ }^{88}$. In contrast, in situ measurements are coupled with the generation process in which a single-coloured fundamental driving laser pulse is assumed. The temporal measurement is insensitive to additional dispersion after generation. Thus, in situ measurements are suitable for studying the dynamics in the generation medium, such as performing molecular tomography ${ }^{31}$, investigating ultrafast molecular rearrangement ${ }^{89}$ and hole dynamics ${ }^{50}$ and resolving the tunnelling time ${ }^{69}$.

\section{Conclusions}

To date, attosecond measurements have mainly used strong-field concepts similar to those that underlie attosecond pulse generation itself. These attosecond-infrared methods have given attosecond science many early successes, including orbital imaging, resolution of attosecond hole dynamics excited by tunnelling and measurement of the time delays in ionization. The similarities and differences between ex situ and in situ measurements provide flexibility. This diversity of approaches to measurement is one of the strengths of attosecond science.

There is no reason why we cannot enhance this diversity by adapting perturbative nonlinear methods to attosecond problems. A promising area for future progress in attosecond science is correlated electronic dynamics. Strongly coupled electronic systems are currently poorly understood. Progress has been made through studying analogous problems involving the atoms in complex molecules using two-dimensional spectroscopy ${ }^{90}$ - a perturbative, three (or more) pulse-pump-probe method. Two-dimensional spectroscopy performed by exciting a molecule at one frequency and observing the influence of this excitation at another frequency has revealed coupling between vibrational modes. By analogy, it should be possible to excite a multi-electron system at one frequency and observe the reaction at another during the exchange of electronic energy in the electronic system ${ }^{91}$.

Received 8 November 2013; accepted 22 January 2014; published online 28 February 2014

\section{References}

1. Cao, J., Ihee, H. \& Zewail, A. H. Ultrafast electron diffraction and direct observation of transient structures in a chemical reaction. Proc. Natl Acad. Sci. 96, 338-342 (1999).

2. Siwick, B. J., Dwyer, J. R., Jordan, R. E. \& Miller, R. J. D. An atomic-level view of melting using femtosecond electron diffraction. Science 302, 1382-1385 (2003).

3. Zewail, A. H. Femtochemistry: Ultrafast Dynamics of the Chemical Bond (World Scientific, 1994)

4. Ihee, H. et al. Direct imaging of transient molecular structures with ultrafast diffraction. Science 291, 458-462 (2001).
5. Chapman, H. N. et al. Femtosecond X-ray protein nanocrystallography. Nature 470, 73-77 (2011).

6. Zhao, K. et al. Tailoring a 67 attosecond pulse through advantageous phasemismatch. Opt. Lett. 37, 3891-3893 (2012).

7. Corkum, P. B. \& Krausz, F. Attosecond science. Nature Phys. 3, 381-387 (2007)

8. Bucksbaum, P. H. The future of attosecond spectroscopy. Science $\mathbf{3 1 7}$, 766-769 (2007).

9. Krausz, F. \& Ivanov, M. Y. Attosecond physics. Rev. Mod. Phys. 81, 163-234 (2009).

10. Scrinzi, A., Ivanov, M. Y., Kienberger, R. \& Villeneuve, D. M. Attosecond physics. J. Phys. B 39, R1 (2006).

11. Krause, J. L., Schafer, K. J. \& Kulander, K. C. High-order harmonic generation from atoms and ions in the high intensity regime. Phys. Rev. Lett. 68, 35353538 (1992).

12. Corkum, P. B. Plasma perspective on strong field multiphoton ionization. Phys. Rev. Lett. 71, 1994-1997 (1993).

13. Lewenstein, M., Balcou, P., Ivanov, M. Y., L'Huillier, A. \& Corkum, P. B. Theory of high-harmonic generation by low-frequency laser fields. Phys. Rev. A 49, 2117-2132 (1994).

14. Salières, P., L'Huillier, A. \& Lewenstein, M. Coherence control of high-order harmonics. Phys. Rev. Lett. 74, 3776-3779 (1995).

15. Lewenstein, M., Salières, P. \& L'Huillier, A. Phase of the atomic polarization in high-order harmonic generation. Phys. Rev. A 52, 4747-4754 (1995).

16. Balcou, P., Salières, P., L'Huillier, A. \& Lewenstein, M. Generalized phasematching conditions for high harmonics: the role of field-gradient forces. Phys. Rev. A 55, 3204-3210 (1997).

17. Paul, P. M. et al. Observation of a train of attosecond pulses from high harmonic generation. Science 292, 1689-1692 (2001).

18. Kitzler, M., Milosevic, N., Scrinzi, A., Krausz, F. \& Brabec, T. Quantum theory of attosecond XUV pulse measurement by laser dressed photoionization. Phys. Rev. Lett. 88, 173904 (2002).

19. Mairesse, Y. \& Quéré, F. Frequency-resolved optical gating for complete reconstruction of attosecond bursts. Phys. Rev. A 71, 011401 (2005).

20. Itatani, J. et al. Attosecond streak camera. Phys. Rev. Lett. 88, 173903 (2002).

21. Sansone, G. et al. Isolated single-cycle attosecond pulses. Science 314, 443-446 (2006).

22. Goulielmakis, E. et al. Single-cycle nonlinear optics. Science 320, 1614-1617 (2008).

23. Quéré, F., Mairesse, Y. \& Itatani, J. Temporal characterization of attosecond XUV fields. J. Mod. Opt. 52, 339-360 (2005).

24. Mairesse, Y. et al. Attosecond synchronization of high-harmonic soft X-rays. Science 302, 1540-1543 (2003).

25. Kim, K. T., Kim, C. M., Baik, M.-G., Umesh, G. \& Nam, C. H. Single sub-50attosecond pulse generation from chirp-compensated harmonic radiation using material dispersion. Phys. Rev. A 69, 051805 (2004).

26. López-Martens, R. et al. Amplitude and phase control of attosecond light pulses. Phys. Rev. Lett. 94, 033001 (2005).

27. Lewenstein, M., Balcou, P., Ivanov, M. Y., L'Huillier, A. \& Corkum, P. B. Theory of high-harmonic generation by low-frequency laser fields. Phys. Rev. A 49, 2117-2132 (1994).

28. Burnett, K., Reed, V. C., Cooper, J. \& Knight, P. L. Calculation of the background emitted during high-harmonic generation. Phys. Rev. A 45, 3347-3349 (1992).

29. Dudovich, N. et al. Measuring and controlling the birth of attosecond XUV pulses. Nature Phys. 2, 781-786 (2006).

30. Kim, K. T. et al. Manipulation of quantum paths for space-time characterization of attosecond pulses. Nature Phys. 9, 159-163 (2013).

31. Itatani, J. et al. Tomographic imaging of molecular orbitals. Nature 432 , 867-871 (2004)

32. Le, A.-T., Lucchese, R. R., Tonzani, S., Morishita, T. \& Lin, C. D. Quantitative rescattering theory for high-order harmonic generation from molecules. Phys. Rev. A 80, 013401 (2009).

33. Frolov, M. V. et al. Analytic description of the high-energy plateau in harmonic generation by atoms: can the harmonic power increase with increasing laser wavelengths? Phys. Rev. Lett. 102, 243901 (2009).

34. Frolov, M. V., Manakov, N. L., Sarantseva, T. S. \& Starace, A. F. Analytic formulae for high harmonic generation. J. Phys. B 42, 035601 (2009).

35. Le, A.-T., Morishita, T. \& Lin, C. D. Extraction of the species-dependent dipole amplitude and phase from high-order harmonic spectra in rare-gas atoms. Phys. Rev. A 78, 023814 (2008).

36. Le, A.-T., Lucchese, R. R., Tonzani, S., Morishita, T. \& Lin, C. D. Quantitative rescattering theory for high-order harmonic generation from molecules. Phys. Rev. A 80, 013401 (2009).

37. Le, V.-H., Le, A.-T., Xie, R.-H. \& Lin, C. D. Theoretical analysis of dynamic chemical imaging with lasers using high-order harmonic generation. Phys. Rev. A 76, 013414 (2007). 
38. Le, A.-T., Lucchese, R. R. \& Lin, C. D. Quantitative rescattering theory of highorder harmonic generation for polyatomic molecules. Phys. Rev. A 87, 063406 (2013)

39. Starace, A. F. Theory of atomic photoionization. In Mehlhorn, W. (ed.) Handbuch Der Physik 31, 1-121 (Springer, 1982).

40. Hüfner, S. Photoelectron Spectroscopy: Principles and Applications (Springer, 2003)

41. Ammosov, M. V., Delone, N. B. \& Krainov, V. P. Tunnel ionization of complex atoms and of atomic ions in an alternating electromagnetic field. Sov. Phys. JETP 91, 2008 (1986).

42. Torres, R. et al. Probing orbital structure of polyatomic molecules by highorder harmonic generation. Phys. Rev. Lett. 98, 203007 (2007).

43. McFarland, B. K., Farrell, J. P., Bucksbaum, P. H. \& Gühr, M. High harmonic generation from multiple orbitals in $\mathrm{N}_{2}$. Science 322, 1232-1235 (2008).

44. Vozzi, C. et al. High harmonic generation spectroscopy of hydrocarbons. App. Phys. Lett. 97, 241103 (2010).

45. Vozzi, C. et al. Generalized molecular orbital tomography. Nature Phys. 7, 822-826 (2011).

46. Li, W. et al. Time-resolved dynamics in $\mathrm{N}_{2} \mathrm{O}_{4}$ probed using high harmonic generation. Science 322, 1207-1211 (2008).

47. Rosca-Pruna, F. \& Vrakking, M. J. J. Experimental observation of revival structures in picosecond laser-induced alignment of $\mathrm{I}_{2}$. Phys. Rev. Lett. 87, $153902(2001)$.

48. Stapelfeldt, H. \& Seideman, T. Colloquium: Aligning molecules with strong laser pulses. Rev. Mod. Phys. 75, 543-557 (2003).

49. Lein, M., Corso, P. P., Marangos, J. P. \& Knight, P. L. Orientation dependence of high-order harmonic generation in molecules. Phys. Rev. A 67, 023819 (2003).

50. Smirnova, O. et al. High harmonic interferometry of multi-electron dynamics in molecules. Nature 460, 972-977 (2009).

51. Zhou, X. et al. Elliptically polarized high-order harmonic emission from molecules in linearly polarized laser fields. Phys. Rev. Lett. 102, 073902 (2009).

52. Shiner, A. D. et al. Probing collective multi-electron dynamics in xenon with high-harmonic spectroscopy. Nature Phys. 7, 464-467 (2011)

53. Kutzner, M., Radojević, V. \& Kelly, H. P. Extended photoionization calculation for xenon. Phys. Rev. A 40, 5052-5057 (1989).

54. Becker, U. et al. Subshell photoionization of Xe between 40 and 1000 eV. Phys. Rev. A 39, 3902-3911 (1989).

55. Fahlman, A., Krause, M. O., Carlson, T. A. \& Svensson, A. Xe 5s, $5 p$ correlation satellites in the region of strong interchannel interactions, $28-75 \mathrm{eV}$. Phys. Rev. A 30, 812-819 (1984).

56. McPherson, A. et al. Studies of multiphoton production of vacuum-ultraviolet radiation in the rare gases. J. Opt. Soc. Am. B 4, 595-601 (1987).

57. Antoine, P., LHuillier, A. \& Lewenstein, M. Attosecond pulse trains using highorder harmonics. Phys. Rev. Lett. 77, 1234-1237 (1996).

58. Christov, I. P., Murnane, M. M. \& Kapteyn, H. C. High-harmonic generation of attosecond pulses in the "single-cycle" regime. Phys. Rev. Lett. 78, 1251-1254 (1997).

59. Corkum, P. B., Burnett, N. H. \& Ivanov, M. Y. Subfemtosecond pulses. Opt. Lett. 19, 1870-1872 (1994).

60. Chini, M., Gilbertson, S., Khan, S. D. \& Chang, Z. Characterizing ultrabroadband attosecond lasers. Opt. Express 18, 13006-13016 (2010).

61. Kienberger, R. et al. Atomic transient recorder. Nature 427, 817-821 (2004).

62. Goulielmakis, E. et al. Direct measurement of light waves. Science 305, 1267-1269 (2004).

63. Wirth, A. et al. Synthesized light transients. Science 334, 195-200 (2011)

64. Schultze, M. et al. Delay in photoemission. Science 328, 1658-1662 (2010).

65. Kheifets, A. S. \& Ivanov, I. A. Delay in atomic photoionization. Phys. Rev. Lett. 105, $233002(2010)$.

66. Kim, K. T., Ko, D. H., Park, J., Tosa, V. \& Nam, C. H. Complete temporal reconstruction of attosecond high-harmonic pulse trains. New J. Phys. 12, 083019 (2010)

67. Doumy, G. et al. Attosecond synchronization of high-order harmonics from midinfrared drivers. Phys. Rev. Lett. 102, 093002 (2009).

68. Kim, K. T. et al. Petahertz optical oscilloscope. Nature Photon. 7, 958-962 (2013).
69. Shafir, D. et al. Resolving the time when an electron exits a tunnelling barrier Nature 485, 343-346 (2012).

70. Véniard, V., Taïeb, R. \& Maquet, A. Phase dependence of $(\mathrm{N}+1)$-color $(\mathrm{N}>1)$ ir-uv photoionization of atoms with higher harmonics. Phys. Rev. A $\mathbf{5 4}$ 721-728 (1996)

71. Jones, D. J. et al. Carrier-envelope phase control of femtosecond mode-locked lasers and direct optical frequency synthesis. Science 288, 635-639 (2000).

72. Baltuška, A. et al. Attosecond control of electronic processes by intense light fields. Nature 421, 611-615 (2003).

73. Mashiko, H. et al. Double optical gating of high-order harmonic generation with carrier-envelope phase stabilized lasers. Phys. Rev. Lett. 100, 103906 (2008)

74. Abel, M. J. et al. Isolated attosecond pulses from ionization gating of highharmonic emission. Chem. Phys. 366, 9-14 (2009).

75. Ferrari, F. et al. High-energy isolated attosecond pulses generated by abovesaturation few-cycle fields. Nature Photon. 4, 875-879 (2010).

76. Wheeler, J. A. et al. Attosecond lighthouses from plasma mirrors. Nature Photon. 6, 829-833 (2012).

77. Kim, K. T. et al. Photonic streaking of attosecond pulse trains. Nature Photon 7, 651-656 (2013).

78. Trebino, R. et al. Measuring ultrashort laser pulses in the time-frequency domain using frequency-resolved optical gating. Rev. Sci. Instrum. 68, 3277-3295 (1997).

79. Iaconis, C. \& Walmsley, I. A. Spectral phase interferometry for direct electricfield reconstruction of ultrashort optical pulses. Opt. Lett. 23, 792-794 (1998).

80. Yakovlev, V. S., Gagnon, J., Karpowicz, N. \& Krausz, F. Attosecond streaking enables the measurement of quantum phase. Phys. Rev. Lett. 105, 073001 (2010).

81. Zhang, C.-H. \& Thumm, U. Streaking and Wigner time delays in photoemission from atoms and surfaces. Phys. Rev. A 84, 033401 (2011).

82. Ivanov, M. \& Smirnova, O. How accurate is the attosecond streak camera? Phys Rev. Lett. 107, 213605 (2011)

83. Dahlström, J. M. et al. Theory of attosecond delays in laser-assisted photoionization. Chem. Phys. 414, 53-64 (2013).

84. Dahlström, J., L'Huillier, A. \& Mauritsson, J. Quantum mechanical approach to probing the birth of attosecond pulses using a two-colour field. J. Phys. B 44, 095602 (2011).

85. Huang, S.-W. et al. High-energy pulse synthesis with sub-cycle waveform control for strong-field physics. Nature Photon. 5, 475-479 (2011).

86. Drescher, M. et al. Time-resolved atomic inner-shell spectroscopy. Nature 419, 803-807 (2002).

87. Cavalieri, A. L. et al. Attosecond spectroscopy in condensed matter. Nature 449, 1029-1032 (2007).

88. Uiberacker, M. et al. Attosecond real-time observation of electron tunnelling in atoms. Nature 446, 627-632 (2007)

89. Baker, S. et al. Probing proton dynamics in molecules on an attosecond time scale. Science 312, 424-427 (2006).

90. Mukamel, S. Multidimensional femtosecond correlation spectroscopies of electronic and vibrational excitations. Annu. Rev. Phys. Chem. 51, 691-729 (2000).

91. Mukamel, S., Healion, D., Zhang, Y. \& Biggs, J. D. Multidimensional attosecond resonant X-ray spectroscopy of molecules: lessons from the optical regime. Annu. Rev. Phys. Chem. 64, 101-127 (2013).

\section{Acknowledgements}

The authors gratefully acknowledge funding from NRC, AFOSR, NSERC and CFI.

\section{Additional information}

Reprints and permissions information is available online at www.nature.com/reprints.

Correspondence and requests for materials should be addressed to P.B.C.

\section{Competing financial interests}

The authors declare no competing financial interests. 